\title{
Myofibrillogenesis regulator-1 promotes cell adhesion and migration in human hepatoma cells
}

\author{
ZHAO WuLi ${ }^{\dagger}$, HE HongWei ${ }^{\dagger}$, REN KaiHuan, ZHANG Hao, CHEN Yi \& SHAO RongGuang* \\ Ministry of Health Key Laboratory of Antibiotic Bioengineering, Department of Oncology, Institute of Medicinal Biotechnology, Peking Union \\ Medical College, Chinese Academy of Medical Sciences, Beijing 100050, China
}

Received November 7, 2012; accepted January 14, 2013; published online June 17, 2013

\begin{abstract}
Myofibrillogenesis regulator-1 $(M R-1)$ is a gene overexpressed usually in many human cancers. However, the effects of MR-1 on cell proliferation, adhesion, migration and genome-wide gene regulation are still unclear. In this study, a human hepatoma cell line that highly overexpresses MR-1, BEL-7402/MR-1 cells was established. While the high expression of MR-1 did not promote cell proliferation, it significantly increased cell spreading, adhesion and migration compared with control cells. A total of 147 genes were regulated by MR-1 expression, 46 genes were down-regulated and 101 genes were up-regulated by MR-1 overexpression. Many of these genes were related to cell adhesion, cytoskeletal regulation, MAPK signaling, and cell cycle related pathways. Western blot analysis further confirmed the regulation of pathways associated with migration by MR-1. These results suggest that MR-1 is involved in the regulation of cancer cell adhesion, migration and related gene expression.
\end{abstract}

MR-1, proliferation, adhesion, migration, gene microarray

Citation: Zhao W L, He H W, Ren K H, et al. Myofibrillogenesis regulator-1 promotes cell adhesion and migration in human hepatoma cells. Chin Sci Bull, 2013, 58: 3007-3014, doi: 10.1007/s11434-013-5933-9

Cancer metastasis is a highly complex process involving several steps: (1) loss of cellular adhesion, (2) increased motility and invasiveness, (3) intravasation and survival in the circulation, (4) extravasation into new tissue, and (5) eventual colonization of the distal site $[1,2]$. Cancer cell migration plays a key role in tumor metastasis and involves several different processes that are regulated by various signaling molecules [3]. Recent advances in the molecular profiling of cancer using genomic-level approaches have revealed many metastasis-related genes, and prompted a reconsideration of how, where, and when cancer cells acquire genes relevant to metastasis [4].

Myofibrillogenesis regulator-1 $(M R-1)$, a new gene involved in metastasis, maps to $2 \mathrm{q} 35$ and was first cloned from a human cDNA library (GenBank accession number AF417001) [5,6]. MR-1 is overexpressed in many human cancers including liver, breast, colon, fibrosarcoma, lung,

$\dagger$ These authors contributed equally to this work.

*Corresponding author (email: shaor@bbn.cn) ovarian [7] and lymphoma (data not published). Chemotherapeutic treatment has been shown to repress the expression of MR-1 in cancer cells [7]. Further, knockdown of MR-1 by siRNA in hepatic carcinoma HepG2 cells decreased cell proliferation, adhesion and invasion [8]. Some of the genes and signaling pathways regulated by MR-1 have been identified, such as Myosin light chain 2 (MLC-2), MAPK, FAK/Akt pathway [8], and NF-кB [9]. However, the gene expression profiles on a genomic level have yet to be determined.

Previously, we have knocked down MR-1 in human hepatoma HepG2 cells using RNAi and found that cell proliferation and migration were blocked both in vitro and in vivo. MR-1 was found to activate MLC-2 though phosphorylation of Ser-19, subsequently stimulating stress fiber formation and activation of the FAK/Akt signaling pathway [8]. It has also been shown that knockdown of MR-1 inhibited cell proliferation in another human hepatoma cell line, BEL-7402 [10].

In this study, we have used BEL-7402 cells and estab- 
lished a stable cell line overexpressing MR-1 (BEL-7402/ MR-1). We have investigated the effect of high MR-1 expression on cell adhesion and migration. Furthermore, we performed a microarray assay to analyze changes in gene expression profiles caused by MR-1 overexpression or knockdown to better understand the molecular alterations associated with MR-1 activity.

\section{Materials and methods}

\subsection{Cell culture and reagents}

Human hepatoma BEL-7402 cells were purchased from the Institute of Biochemistry and Cell Biology (Shanghai, China), and grown in RPMI-1640 (Hyclone, Utah, USA) supplemented with $10 \%$ fetal bovine serum (FBS) (Tianjing Hao Hai Biomaterial Technology Company, China), penicillin-streptomycin $(100 \mathrm{U} / \mathrm{mL})$, and $2 \mathrm{mmol} / \mathrm{L} L$-glutamine. Lipofectamine ${ }^{\mathrm{TM}} 2000$ and RNAiMAX were from Invitrogen Life Technologies (Carlsbad, CA, USA), anti- $\beta$-actin (sc-1616), horseradish peroxidase-conjugated goat anti-rabbit (ZB-2301) or rabbit anti-goat (ZB-2306) secondary antibodies were from Santa Cruz Biotechnologies (Santa Cruz, CA, USA). Rabbit anti-MR-1 polyclonal antibody was a kind gift from Dr. Yiguang Wang. Fibronectin, Cy3- dCTP and Cy5-dCTP were from Pharmacia (Stockholm, Sweden). Twenty-two thousand human genomic microarrays were from CapitalBio (Beijing, China).

\subsection{Plasmid construction}

DNA primers for $M R-1$ were synthesized by Sai Bai Sheng Co. (Beijing, China) as follows; MR1a: 5'-ATTAGGATCCATGGCGGCGGTGGTAGCTGCT-3' and MR1b: 5'-ATTAGAATTCTCAGGTCTGCACCCCAGACCC-3'. These primers were used to amplify full-length $M R-1$ from human hepatoma HepG2 cells using PCR. The amplified product was the subcloned into the BamHI-EcoRI sites in pCDNA3.1 (ClonTech, CA, USA).

\subsection{Plasmid transfection}

Cells were cultured in 10\% FBS medium without antibiotics 18-20 h before transfection. Once cells reached $90 \%$ confluency, they were transfected with either MR-1 or vector alone (to generate the negative control BEL-7402/EV cells) with Lipofectamine 2000 according to the manufacturer's instructions. Six hours after transfection, the medium was replaced using $10 \%$ FBS medium without antibiotics.

\subsection{SiRNA and transfection}

siRNAs were bought from RiBoBio Co. (Guangzhou, China) with the following sequences: mock-siRNA: 5'-UUCUCCGAACGUGUCACGUdTdT-3' and MR-1-siRNA: 5'-CCU-
AGGCUAUUGACUGUUAdTdT-3'. Cells were trypsinized and seeded to 6- well plate with appropriate concentration in fresh medium without antibiotics. After 18-20 h, when reached 30\%-50\% confluency. Cells were transfected with the siRNAs using Lipofectamine RNAiMAX according to the manufacturer's instructions.

\subsection{Stable transfection of MR-1}

After transfected with pCDNA3.1-MR-1 for $24 \mathrm{~h}$, the cells were cultured in selection media (containing $400 \mu \mathrm{g} / \mathrm{mL}$ G418) for 2 weeks until all non-transfected cells had died. The remaining cells were then trypsinized, counted, and diluted to 5 cells $/ \mathrm{mL}$ in a volume of $100 \mu \mathrm{L}$ per well in 96-well plate. Those wells that contained only one cell were cultured until confluent, and then transferred sequentially to a 24-well plate, a 6-well plate and finally a $25-\mathrm{mL}$ flask.

\subsection{Cell proliferation and colony formation}

To measure cell proliferation, cells were seeded at a density of 1000 per well in 24-well plates. The medium was changed daily, and cells were counted every $24 \mathrm{~h}$ for $7 \mathrm{~d}$. To assay colony formation, cells were seeded at a density of 100 per well in 24-well plates. Culture medium was changed every $4 \mathrm{~d}$ and colonies were stained with hematoxylin, photographed and counted after $7 \mathrm{~d}$. Colony formation=number of colonies/number of cells seeded $\times 100 \%$.

\subsection{Western blotting}

Transfected cells were lysed in $100 \mu \mathrm{L}$ RIPA lysis buffer (50 mmol/L Tris-HCl, pH 7.5, $150 \mathrm{mmol} / \mathrm{L} \mathrm{NaCl}, 1 \%$ $\mathrm{NP}-40,1 \mathrm{mg} / \mathrm{mL}$ leupeptin, $1 \mathrm{mg} / \mathrm{mL}$ aprotinin, $1 \mathrm{mmol} / \mathrm{L}$ $\mathrm{NaF}, 1 \mathrm{mmol} / \mathrm{L} \mathrm{Na}_{3} \mathrm{VO}_{4}$ ) at $4^{\circ} \mathrm{C}$ for $30 \mathrm{~min}$. Then centrifuged the tubes at $12000 \times \mathrm{g}$ for $20 \mathrm{~min}$ at $4^{\circ} \mathrm{C}$ and saved the supernatant. Protein concentration was determined by the Bradford assay. Equal amount of lysate $(40 \mu \mathrm{g})$ was used to run SDS-polyacrylamide gel and transferred to polyvinylidene fluoride (PVDF) membrane (Millipore, Bedford, MA, USA). Then block the membranes with $5 \%$ non-fat milk in TBS-T buffer (20 mmol/L Tris- $\mathrm{HCl}, \mathrm{NaCl} 0.9 \%(\mathrm{w} / \mathrm{v}), 0.1 \%$ Tween-20) at $25^{\circ} \mathrm{C}$ for $1 \mathrm{~h}$ and incubated with primary antibodies for $2 \mathrm{~h}$. Appropriate horseradish peroxidase-linked secondary antibodys were then used for $1 \mathrm{~h}$ membrane incubation. After that the membranes were incubate with ECL reagents (Millipore) and took photoes with a ChemiImager 5500 imaging system (Alpha Innotech Corporation, San Leandro, CA, USA).

\subsection{Cell adhesion assay}

A 96-well plate was coated with $10 \mu \mathrm{g} / \mathrm{mL}$ fibronectin (FN)(MERKE, Germany) for $1 \mathrm{~h}$ and then blocked with $1 \mu \mathrm{g} / \mathrm{mL}$ bovine serum albumin (BSA) for $1 \mathrm{~h}$. Cells were 
trypsinized, counted, and washed in serum-free RPMI1640 medium and then resuspended in culture medium. Then 100 $\mu \mathrm{L}$ of suspended cells was added to each well of the wells and then incubated for $15,30,60$ or $90 \mathrm{~min}$ at $37^{\circ} \mathrm{C}$ in a $\mathrm{CO}_{2}$ incubator. Cells that had been attached for $10 \mathrm{~h}$ were used as a negative control. Attached cells were analyzed by MTT assay after non-adherent cells were removed by washing with PBS as described previously [8]. Adhesion rate $=\left(A_{570}\right.$ adherent cells $/ A_{570}$ control cells $) \times 100 \%$.

\subsection{Analysis of cell spreading morphology}

Cells were trypsinized, counted, suspended in serum-free RPMI1640 medium, and then seeded on $10 \mu \mathrm{g} / \mathrm{mL}$ FNcoated 96 -well plates for 30,60 and $120 \mathrm{~min}$ at $37^{\circ} \mathrm{C}$. They were then photographed using a Nikon upright scope equipped with a camera (Eclipse TE2000-U, Nikon, Japan).

\subsection{Wound-healing and cell migration assays}

Cells were seeded in 24-well plates and grown in growth medium to near confluence, and carefully draw a linear "wound" in the cell monolayer of each well using a plastic pipette tip scratched. Then washed with phosphate-buffered saline (PBS) twicely to remove detached cells and debris from the monolayer. The plates were monitored by an Eclipse TE2000-U image analysis system after 24 and $48 \mathrm{~h}$. The wounded area was measured and quantified (Image Pro-Plus software) as wound closure and reported as percentage wound healing using the following equation: \% wound healing $=\left[1-\left(\right.\right.$ wound area at $t_{24 / 48} \mathrm{~h} /$ wound area at $\left.\left.t_{0}\right)\right]$ $\times 100$, where $t_{0}$ is the time immediately after wounding. For cell migration assays, cells were suspended in serum-free RPMI1640 medium containing 0.1\% BSA were applied to the upper chamber of a Transwell plate (Costar, Cambridge, MA, USA). RPMI1640 containing 20\% FBS and $10 \mu \mathrm{g} / \mathrm{mL}$ FN was added to the lower chamber. After incubation at $37^{\circ} \mathrm{C}$ for $3 \mathrm{~h}$, cells that had migrated to the lower chamber and were stained with hematoxylin, and counted as cells per microscopic field (400× magnification).

\subsection{RNA extraction and microarray analysis}

Total RNA was extracted using TRIzol reagent (Invitrogen) and purified with NucleoSpin RNA clean-up kit (Macherey-Nagel, Germany). RNA were labeled with $\mathrm{Cy} 3$ and Cy5 using a cRNA labeling method following the protocol from CapitalBio (Beijing, China). Briefly, the double-stranded cDNAs were synthesized using T7-Oligo $(\mathrm{dT})_{15}$ (5'-AAACGACGGCCAGTGAATTGTAATACGACTCACTATAGGCGCTTTTTTTTTTTTTTTTG-3') primers. cRNA was then generated by in vitro transcription. Reverse transcription was then performed using nine base (N9) random primers. The products of the reverse transcription reaction were labeled with $\mathrm{Cy} 3$ and $\mathrm{Cy} 5$ using KLENOW and N9 random primers. Fluorescence exchange was done to eliminate false positive and negative signals. Labeled DNA was hybridized with the microarrays at $42^{\circ} \mathrm{C}$ overnight. After hybridization and subsequent washing, the arrays were analyzed with a LuxScan 10K/A dual channel laser scanner (CapitalBio). Data were normalized using Lowess method, and only those genes with consistent alteration tendency (both $>1.5$-fold) in both microarrays were choosed as differentially expressed genes. Gene function and pathway analysis was finished by Pathway Miner (http://www.biorag.org/) and DAVID Bioinformatics Resources (The Database for Annotation, Visualization and Integrated Discovery).

\section{Results}

\subsection{Establishment of MR-1 stable high-expressing cells BEL-7402/MR-1}

MR-1 was successfully cloned into the plasmid pcDNA3.1 and named pcDNA3.1-MR-1. This vector could generate full-length MR-1 protein stably and effectively. After stable transfection of pcDNA3.1-MR-1 into human hepatocellular carcinoma BEL-7402 cells, the levels of MR-1 were significantly increased (Figure 1). Stably transfected clone \#1 was selected and named BEL-7402/MR-1 for the remaining experiments. Another clone from cells stably transfected with the control vector pcDNA3.1 was randomly selected and named BEL-7402/EV as a negative control.

\subsection{Overexpression of MR-1 did not promote cell pro- liferation}

Cell growth curves and colony formation assays were used to analyze the ability of MR-1 to regulate cell growth and colony formation of BEL-7402 cells. We found no significant difference in cell growth or colony formation among BEL-7402, BEL-7402/EV and BEL-7402/MR-1 cells (Figure 2).

\subsection{MR-1 increased cell spreading}

To test cell spreading efficiency, BEL-7402, BEL-7402/EV and BEL-7402/MR-1 cells were plated on FN-coated cell culture dishes (Figure 3). BEL-7402/MR-1 cells began to spread on FN-coated culture plates 60 min after seeding,

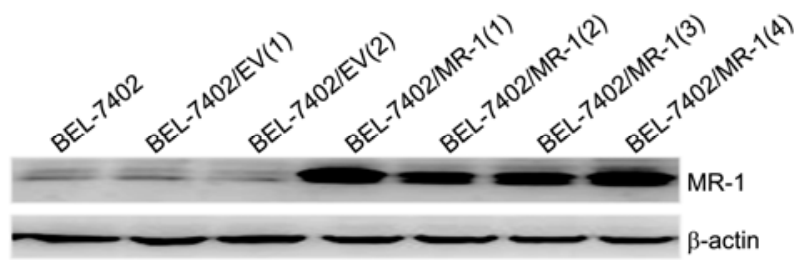

Figure 1 MR-1 was highly expressed in human hepatoma BEL-7402/ MR-1 cells as detected by Western blot analysis. 

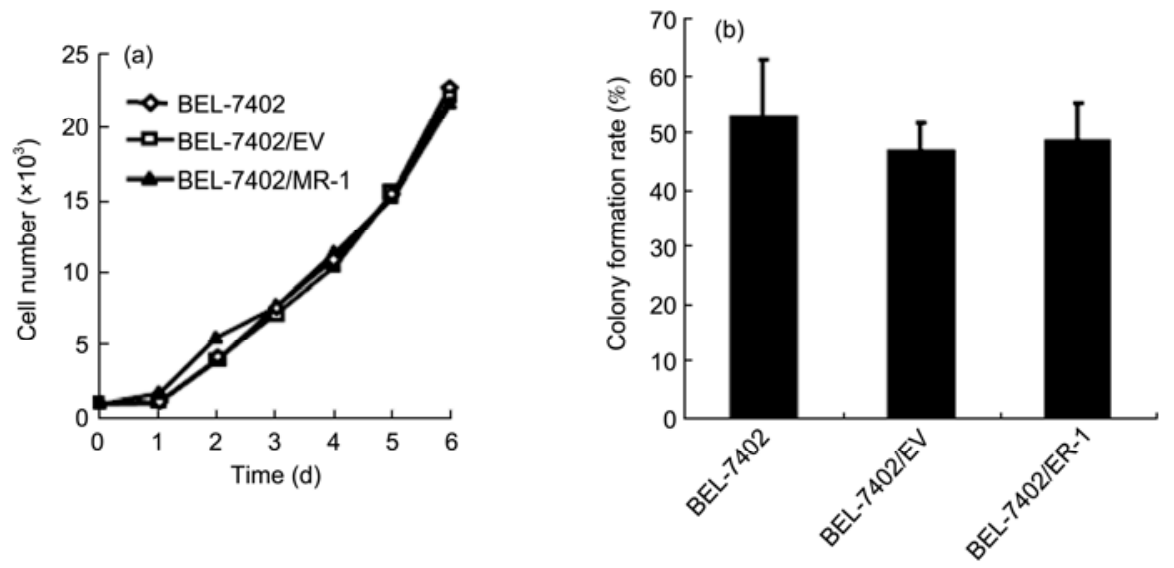

Figure 2 MR-1 did not significantly affect cell growth (a) and colony formation (b).
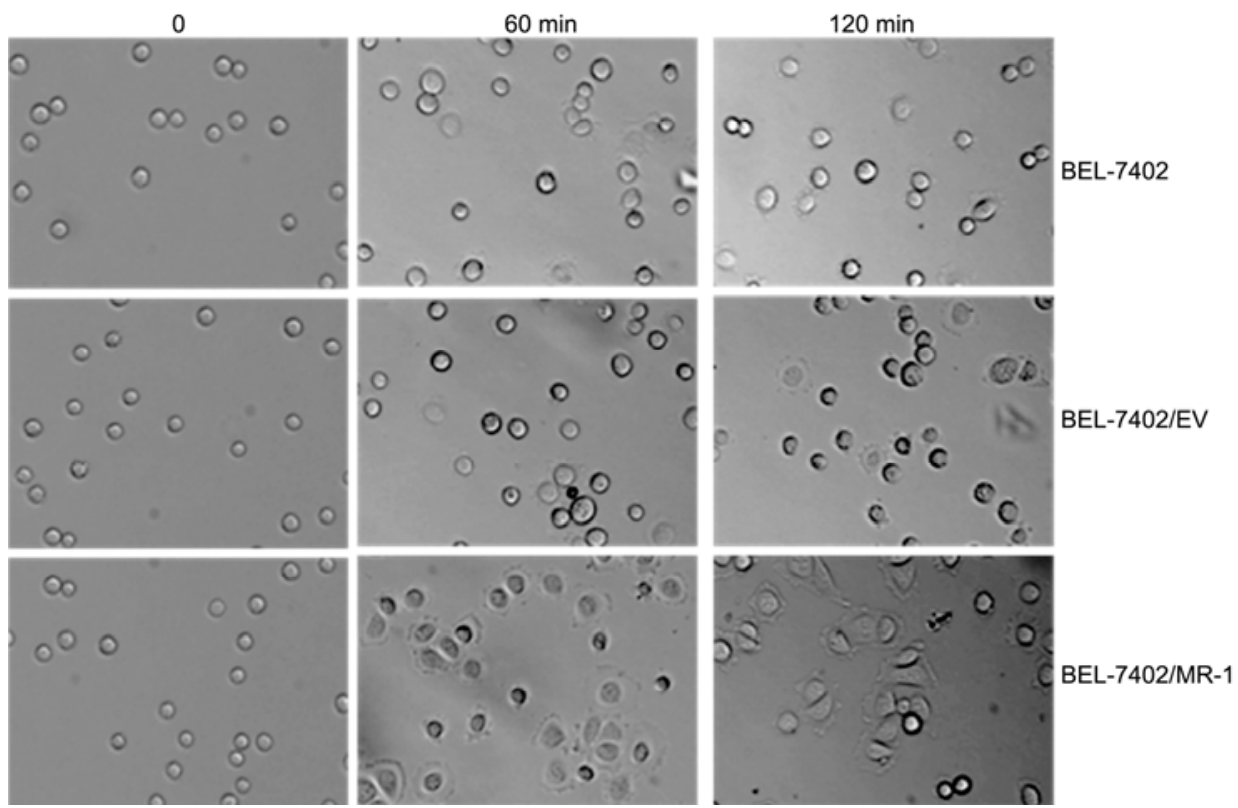

Figure 3 Cell spreading was increased in BEL-7402/MR-1 cells.

while most of the BEL-7402 and BEL-7402/EV cells still remained rounded. After $120 \mathrm{~min}$, most of the BEL-7402/ MR-1 cells were much better spread compared with BEL7402 and BEL-7402/EV cells. These data indicated that MR-1 could enhance the spreading ability of BEL-7402 cells.

\subsection{MR-1 increased cell adhesion}

Cell adhesion to the extracellular matrix is a key step in invasion and metastasis. As shown in Figure 4, compare with BEL-7402 and BEL-7402/EV cells, the cell adhesion to $\mathrm{FN}$ of BEL-7402/MR-1 cells was significantly higher.

\subsection{MR-1 increased cell migration}

Wound-healing and Transwell culture assays were used to analyze the ability of MR-1 to regulate cell migration.
Wound-healing assay showed that BEL-7402/MR-1 cells, but not BEL-7402 and BEL-7402/EV cells, began to migrate into the scratched wound after $24 \mathrm{~h}$. After $48 \mathrm{~h}$, BEL7402 /MR-1 cells migration into the scratched area was much more evident compared with BEL-7402/EV and BEL7402 cells. Wound healing analysis showed that after $48 \mathrm{~h}$, BEL-7402/MR-1 cells had covered approximately $80 \%$ of the scratched wound, which is significantly higher than either the BEL-7402/EV (53\%) or BEL-7402 cells (45\%) (Figure 5(a)).

In Transwell culture systems, cells can migrate through a porous membrane when high concentrations of serum are presented as a chemotactic factor. In this assay, BEL-7402/ MR-1 cells showed significantly increased chemotaxis (29.7\% more) compared with BEL-7402 cells (Figure 5(b)) $(P<0.05)$. This indicated that MR-1 could enhance the chemotactic migration of BEL-7402 cells. 


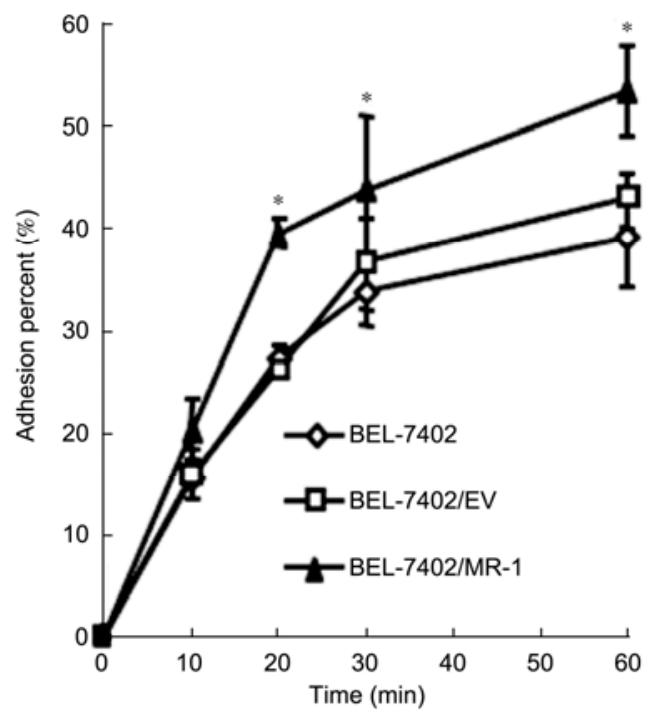

Figure 4 Cell adhesion to FN was increased in BEL-7402/MR-1 cells. $* P<0.05$.

\subsection{MR-1 altered the gene expression profile in BEL- 7402/MR-1 cells}

To analyze the genome-wide alteration gene expression, total RNA was extracted from BEL-7402/EV and BEL-7402/ MR-1 cells for microarray experiments. For our analysis, an alteration of 1.5-fold signal intensity was regarded as a significant change in mRNA expression, and 338 genes were up-regulated and 980 genes were down-regulated in BEL7402/MR-1 cells compared with BEL-7402/EV cells (data not shown).

In addition to analyzing the effects of MR-1 overexpression, we also analyzed the effects of MR-1 knockdown on gene expression in BEL-7402 cells. Genes with significantly changed expression levels were clustered using a hierarchical average linkage clustering program for MR-1 siRNA treated cells (As described previously [8], siRNA efficiently reduced the expression of MR-1, data not shown) (Figure 6). We found a total of 249 genes affected by both MR-1 overexpression and knockdown which we divided into four clusters. In Cluster 1, 101 genes were up-regulated in BEL-7402/MR-1 cells and down-regulated in MR-1 siRNA-treated cells. By contrast, in Cluster 4, we identified 47 genes that were down-regulated in BEL-7402/MR-1 cells and up-regulated in MR-1 siRNA-treated cells.

We used a gene functional enrichment analysis using DAVID Bioinformatics Resources [10,11] to further characterize the genes affected by MR-1 expression (Clusters 1

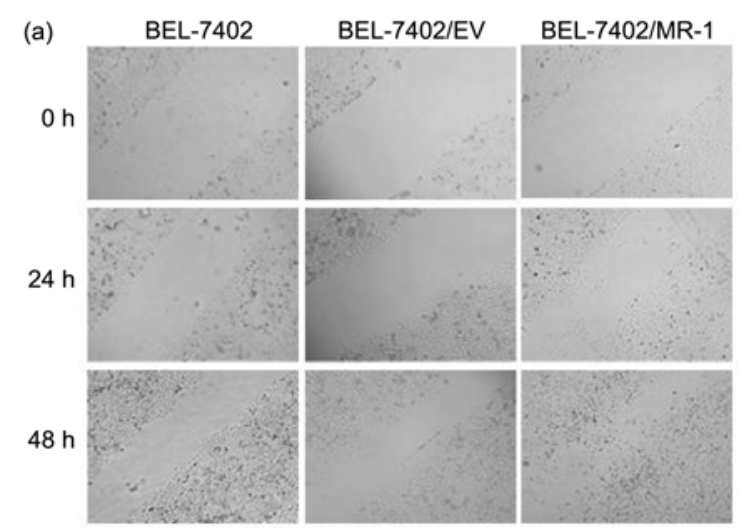

(b)

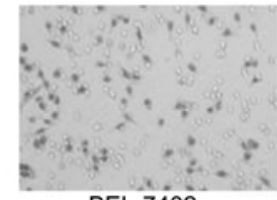

BEL-7402

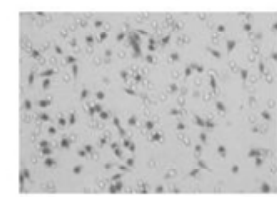

BEL-7402/EV

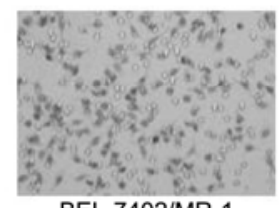

BEL-7402/MR-1

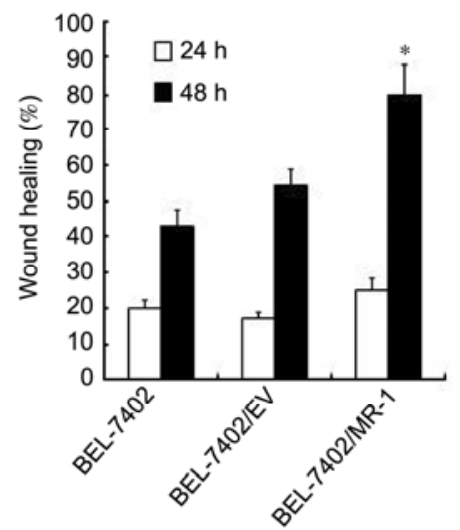

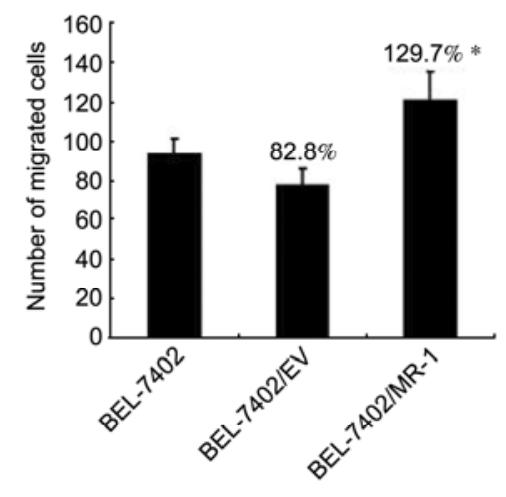

Figure 5 Cell migration was increased in BEL$7402 /$ MR-1 cells as measured by wound-healing (a) and Transwell migration assays (b). Cell numbers were counted under the microscope after hematoxylin staining. $* P<0.05$. 


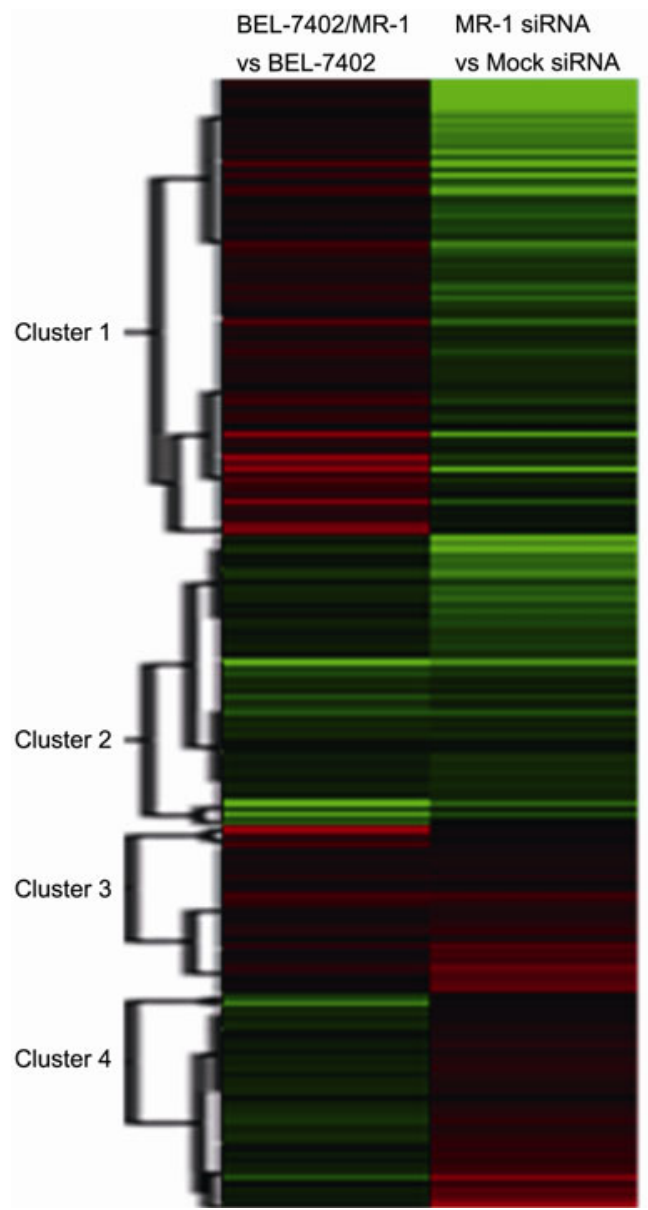

Figure 6 Cluster analysis of gene expression changes measured by microarray analysis. Red: increase in gene expression; green: decrease in gene expression.

and 4). We showed that the up-regulated genes are enriched in tumor-related functions, including cell-cell signaling, cell proliferation, cell differentiation, cell survival, and cell adhesion (Table 1). These genes have also been shown to be enriched by other conditions, including stress and drug response (data not shown).

We choose genes in the Kyoto Encyclopedia of Gene and Genomes (KEGG) database which had higher expression levels and had a crucial role in migration to further validate the protein expression levels of these genes. We demonstrated that the expression levels of FN1, SDC2 and ITGA5 were up-regulated in BEL-7402/MR-1 cells compared with BEL-7402/EV and BEL-7402 cells (Figure 7). This suggests that the enhancement of cell migration in BEL-7402/MR-1 cells might be partly regulated by the up-regulation of these proteins.

Pathway analysis was done using a network platform, Pathway miner. In this platform, after uploading genes and their changed ratio values, their related pathways are indicated. The analysis of signaling pathways by the KEGG database showed that MR-1 affect pathways related to cell adhesion, cytoskeletal regulation, cytokine interactions, and
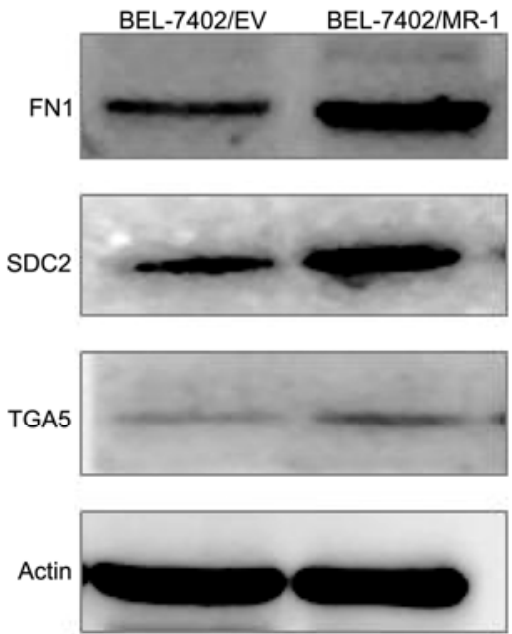

Figure 7 The expression levels of proteins related to migration measured by Western blot analysis.

Table 1 Enrichment analysis of gene function regulated by MR-1

\begin{tabular}{llc}
\hline Gene type affected by MR-1 & Gene function & $P$-value \\
\hline & Cell proliferation & 0.021 \\
& Cell differentiation & 0.022 \\
Up-regulated genes & Cell survival & 0.022 \\
& Cell adhesion & 0.040 \\
& Cell motility & 0.079 \\
\hline Down-regulated genes & Cell cycle & 0.037 \\
\hline
\end{tabular}

cell cycle signal transduction pathway. This analysis indicated that MR-1 might play a role in tumor metastasis and invasion (Table 2), consistent with the above experimental results.

\section{Discussion}

During the process of cancer metastasis, cancer cells invade into the surrounding normal tissue. It was found that prior to invasion; some highly mobile cells with elevated levels of ERK1/2 activity already existed in pre-invasive tumors [12]. Therefore, the molecular control of cell migration may be an important issue for cancer therapy [13]. Tumor cell migration is a complex process and is regulated by many signaling molecules, such as focal adhesion kinase (FAK) that regulates cell-matrix adhesion, and myosin light chain kinase (MLCK) or Rho-related coiled-coil forming protein kinase (ROCK) that regulate cell migration by promoting myosin II contraction [14-16]. MR-1 is a novel protein discovered by Wang and colleagues that can stimulate the phosphorylation of MLC-2, affect cell-matrix adhesion through MLC-2/FAK/Akt pathways, and regulate cell migration and proliferation [8]. To further elucidate the role of MR-1, we generated human hepatoma cells that stably overexpress MR-1, BEL-7402/MR-1 cells. We analyzed the effects of high 
Table 2 MR-1 regulated genes involved signalling pathway in KEGG database

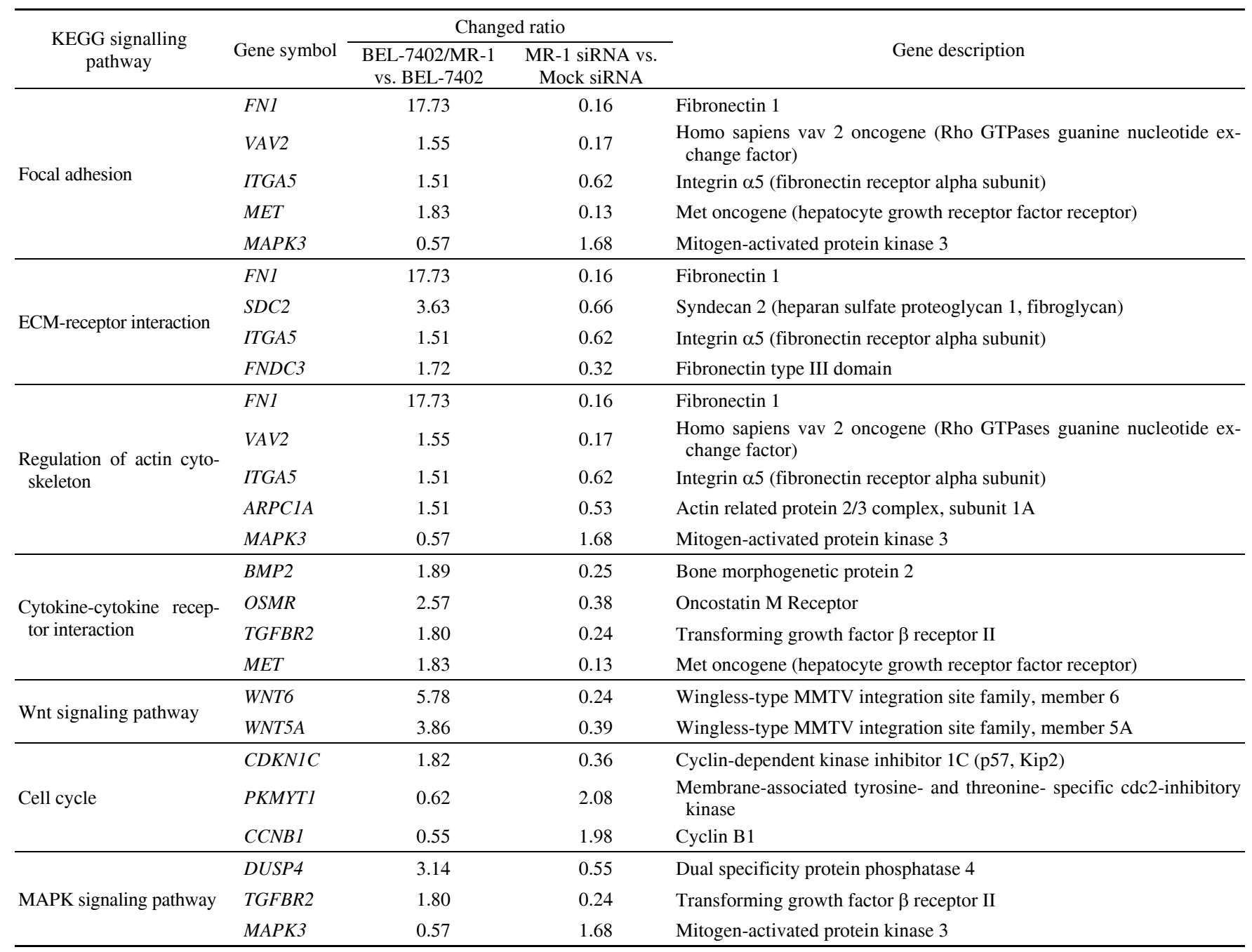

expression of MR-1 on proliferation, colony formation, adhesion, migration as well as genome-wide mRNA expression.

Cell proliferation and colony formation assays showed the proliferative ability of BEL-7402/MR-1 cells was not higher than controls, BEL-7402 and BEL-7402/EV cells. However, previous studies have shown that cell proliferation was significantly reduced after MR-1 was knocked down by RNAi in BEL-7402 and HepG2 cells [10]. This might a result of the complex regulation of signaling pathways related to proliferation-overexpression of MR-1 in cells whose MR-1 level are already highly expressed might trigger negative feedback that represses its proliferative activity. In contrast to proliferation, cell spreading, migration, and adhesion of BEL-7402/MR-1 cells were all significantly increased. Further, these activities were all decreased in response to MR-1 knockdown. These results confirm that MR-1 plays an important role in cell spreading, adhesion and migration in human hepatoma cells.

Tumorigenesis and metastasis are related to numerous macromolecules with very complex interactions. We used a human genome oligonucleotide microarray to analyze global gene expression changes induced by MR-1 overexpression or knockdown. MR-1 expression regulates many biological processes, including cell proliferation, differentiation, and migration.

The regulation of MR-1 expression affected many genes related to cell proliferation, migration and invasion, including MAPK, Wnt, and actin cytoskeleton regulatory proteins. One of the genes that was prominently upregulated in BEL7402/MR-1 cells was fibronectin, FN1. FN1 mRNA levels were increased 17.7-fold and reduced 6.2-fold in MR-1 knocked down cells. Fibronectin is an important extracellular matrix protein that promotes the tyrosine phosphorylation of paxillin and other focal adhesion proteins and can stimulate cancer cell invasion and metastasis [17]. FN1 is now used as a diagnostic marker for several kinds of cancer, such as thyroid cancer and malignant melanoma [18]. VAV2 mRNA level in BEL-7402/MR-1 cells was increased by 1.5-fold, and decreased by 5.6-fold in MR-1 knockdown cells. VAV2 is a guanine nucleotide exchange factor for Rho 
GTPases and can inhibit the internalization and degradation of growth factor receptor. This stimulates the phosphorylation of EGFR, ERK and Akt and promotes growth factor-induced cell adhesion, migration, spreading and proliferation [19]. Another gene with a similar regulation pattern was integrin $\alpha 5$ (ITGA5), a subunit of the $\alpha 5 \beta 1$ integrin that is a specific fibronectin receptor and mediates cell attachment, migration, and proliferation in many cells [20]. SDC2 expression was also regulated by MR-1 and can promote Tiam1-dependent Rac activation to promote migration of rectal cancer cells [21]. ARPCIA, a new tumor- related gene, was also affected by MR-1 expression levels. This is an actin-related protein and has been found to be an important regulator of cell motility and invasion is in pancreatic cancer cells $[22,23]$. Altogether, there were a number of important genes related to cell adhesion; invasion and spreading that were regulated by MR-1 expression. The regulation of these pathways might promote cancer cell invasion and metastasis induced by MR-1 overexpression in tumors.

For genes related to cell proliferation, MR-1 expression showed heterogeneous effects. On the one hand, MR-1 overexpression up-regulated pathways that promotes cell growth, such as MAPK and Wnt signaling. On the other hand, MR-1 overexpression also increased the expression of cyclin-dependent kinase inhibitor 1C (p57) and reduced CCNB1 levels, which represses cell cycle signaling. These non-congruent effects may help explain why the overexpression of MR-1 in BEL-7402 cells showed no significant changes in cell proliferation.

In summary, we have shown that MR-1 may be involved in and regulate tumor cell proliferation, adhesion and migration by regulating a number of signaling pathways involved in these complex processes. Further research will need to clarify the role of MR-1 in these pathways, elucidate its upstream regulation, and identify its direct target genes. Together, this research should help clarify the role of MR-1 in cancer progression as well as its potential as a novel therapeutic target.

This work was supported by the National Basic Research Program of China (2009CB521807), the National Natural Science Foundation of China (30772583, 30900237), and National S\&T Major Special Project on Major New Drug Innovation (2012ZX09301002-001).

1 Chambers A F, Groom A C. MacDonald I C. Dissemination and growth of cancer cells in metastatic sites. Nat Rev Cancer, 2002, 2: 563-572

2 Fidler I J. The pathogenesis of cancer metastasis: The "seed and soil" hypothesis revisited. Nat Rev Cancer, 2003, 3: 453-458

3 Gupta G P, Massague J. Cancer metastasis: Building a framework. Cell, 2006, 127: 679-695

4 Bernards R, Weinberg R A. A progression puzzle. Nature, 2002, 418: 823

5 Wang Y G, Li T B, Feng S. Cell reconstruction, transduction and apoptosis related gene CRI. PRC Patent, CN 02153656.2, 2002

6 Li T B, Liu X H, Feng S, et al. Characterization of MR-1, a novel myofibrillogenesis regulator in human muscle. Acta Biochim Biophys Sin (Shanghai), 2004, 36: 412-418

7 Lu R, Sun M, Feng J, et al. Myofibrillogenesis regulator 1 (MR-1) is a novel biomarker and potential therapeutic target for human ovarian cancer. BMC Cancer, 2011, 11: 270

8 Ren $\mathrm{K}$, Jin $\mathrm{H}$, Bian C, et al. MR-1 modulates proliferation and migration of human hepatoma HepG2 cells through myosin light chains-2 (MLC2)/focal adhesion kinase (FAK)/Akt signaling pathway. J Biol Chem, 2008, 283: 35598-35605

$9 \mathrm{Li} \mathrm{H} \mathrm{L}$, She Z G, Li T B, et al. Overexpression of myofibrillogenesis regulator-1 aggravates cardiac hypertrophy induced by angiotensin II in mice. Hypertension, 2007, 49: 1399-1408

10 Wang J H, Bian $\mathrm{C}$ J, Zhao $\mathrm{C} \mathrm{H}$, et al. MR1 siRNA suppresses proliferation of human hepatoma BEL-7402 cells (in Chinese). Basic Clinical Medicine, 2009, 29: 1-5

11 Zhao M, He H W, Sun H X. Dual knockdown of N-ras and epiregulin synergistically suppressed the growth of human hepatoma cells. Biochem Biophys Res Commun, 2009, 387: 239-244

12 Huang D W, Sherman B T, Lempicki R A. Systematic and integrative analysis of large gene lists using DAVID bioinformatics resources. Nat Protoc, 2009, 4: 44-57

13 Pearson G W, Hunter T. Real-time imaging reveals that noninvasive mammary epithelial acini can contain motile cells. J Cell Biol, 2007, 179: $1555-1567$

14 Yamazaki D, Kurisu S, Takenawa T. Regulation of cancer cell motility through actin reorganization. Cancer Sci, 2005, 96: 379-386

15 Nobes C D, Hall A. Rho GTPases control polarity, protrusion, and adhesion during cell movement. J Cell Biol, 1999, 144: 1235-1244

16 Alblas J, Ulfman L, Hordijk P. Activation of Rhoa and ROCK are essential for detachment of migrating leukocytes. Mol Biol Cell, 2001, 12: 2137-2145

17 Raftopoulou M, Hall A. Cell migration: Rho GTPases lead the way. Dev Biol, 2004. 265: 23-32

$18 \mathrm{Li} \mathrm{D}$, Ding J, Wang X, et al. Fibronectin promotes tyrosine phosphorylation of paxillin and cell invasiveness in the gastric cancer cell line AGS. Tumori, 2009, 95: 769-779

19 Griffith O L, Melck A, Jones S J, et al. Meta-analysis and metareview of thyroid cancer gene expression profiling studies identifies important diagnostic biomarkers. J Clin Oncol, 2006, 24: 5043-5051

20 Thalappilly S, Soubeyran P, Iovanna J L, et al. VAV2 regulates epidermal growth factor receptor endocytosis and degradation. Oncogene, 2010, 29: 2528-2539

21 Gu J, Isaji T, Sato Y, et al. Importance of $N$-glycosylation on alpha5beta1 integrin for its biological functions. Biol Pharm Bull, 2009, 32: 780-785

22 Choi Y, Kim H, Chung H, et al. Syndecan-2 regulates cell migration in colon cancer cells through Tiam1-mediated Rac activation. Biochem Biophys Res Commun, 2010, 391: 921-925

23 Laurila E, Savinainen K, Kuuselo R, et al. Characterization of the 7q21-q22 amplicon identifies ARPC1A, a subunit of the Arp2/3 complex, as a regulator of cell migration and invasion in pancreatic cancer. Genes Chromosomes Cancer, 2009, 48: 330-339

Open Access This article is distributed under the terms of the Creative Commons Attribution License which permits any use, distribution, and reproduction in any medium, provided the original author(s) and source are credited. 